\title{
Sur quelques Ixodoidea (Acarina) paléarctiques inféodés aux micro-Chiroptères
}

\author{
Par J.-C. BEAUCOURNU
}

Nous avons antérieurement (Beaucournu, 1961), au cours d'une étude sur les ectoparasites de Chiroptères dans l'Ouest de la France, mentionné la présence dans cette région de deux Ixodoïđés communs sur ces animaux: Argas vespertilionis (Latreille, 1802) et Ixodes vespertilionis Koch, 1844. Depuis cette époque, nous avons eu l'occasion d'étudier un matériel plus varié en provenance de diverses régions de France ou de divers autres points de la région paléarctique occidentale, prélevé par nous-même ou adressé pour étude par divers collecteurs.

Si l'on excepte des références anciennes telles que celles d'Haemaphysalis rhinolophi Canestrini et Fanzago 1877 ( $=H$. punctata Can. et Fanz.) décrit au siècle dernier en Italie sur Rhinolophus ferrumequinum, puis signalé en Hollande sur Plecotus auritus par Neumann (1897) et qui, depuis, ne semble pas avoir été revu sur des Chiroptères, ou celle de Dermacentor reticulatus (Fabricius, 1794) cité une fois sur chauve-souris (Miniopterus schreibersi) in Neumann 1911 (1), huit Ixodoïdés ont été décrits dans la région paléarctique occidentale comme parasitant normalement les chauves-souris insectivores. Ce sont quatre espèces appartenant au genre Ixodes [I. vespertilionis Koch, 1844 ; I. simplex simplex Neumann 1906; I. chiropterorum Babos et Janisch, 1958 et I. pospelovae Emtchuk, 1955] et quatre espèces du genre Argas [A. (Carios) vespertilionis (Latreille, 1802); A. (Secretargas) transgariepinus White, 1846; A. (Chiropterargas) boueti Roubaud et Colas-Belcour, 1933 et A. (Chiropterargas) confusus Hoogstraal, 1955].

En ce qui concerne les Ixodes, nous apportons de nouveaux points de répartition pour I. vespertilionis et $I$. simplex et nous plaçons les deux autres en synonymie de ce dernier.

Quant aux Argas, les deux Chiropterargas ne sont connus que d'Egypte et de Palestine (2) en ce qui concerne la région paléarctique. Les deux autres ont une répartition beaucoup plus vaste et nous en donnons de nouvelles captures.

(1) Nous pensons d'ailleurs que ces références sont erronées et proviennent d'une confusion de matériel. Les hôtes normaux de ces deux espèces sont, au stade adulte, les ongulés.

(2) $A$. boueti est connu de ces deux pays; $A$. confusus, d'Egypte. 
Pour terminer la liste des Ixodoïdés capturés sur les Chiroptères dans cette zone, nous devons enfin mentionner Ornithodoros coniceps (Canestrini 1890) signalé en Tunisie par Colas-Belcour (1929) sur Pipistrellus kuhli alors que son hôte normal est le pigeon, $O$. salahi Hoogstraal 1953, de Palestine et d'Egypte, parasite de Chiroptères frugivores, et enfin un argasidé, que nous avons récolté à l'état larvaire en Algérie sur Eptesicus et dont nous ne pouvons actuellement préciser davantage le statut (3).

\section{$1^{\circ}$ IXODES VESPERTILIONIS Koch 1844}

Cette tique, cavernicole, est commune dans toute la France (Neumann 1916; Beaucournu 1961). Le très abondant matériel examiné tant mort que vivant nous a permis d'éclaircir quelque peu la biologie et la morphologie de cette espèce. Nous y reviendrons dans une note ultérieure.

Il nous paraît inutile d'énumérer les diverses stations d'où nous avons identifié cette tique : elle est en effet présente dans toute la France continentale (4). Citons toutefois :

Corse :

— Moltifao (17-7-64), grotte de Pietralbello; sur paroi : 1 đ, 1 nymphe (J.C.B. rec.).

En dehors de France, nous avons examiné des exemplaires de:

Suisse :

- (Soleure), grotte aux environs d'Erschwil [S.O. 6] (5) (16-4-61); 1 nymphe gorgée, libre (Roth, Geiser et Strinati rec.).

- (Soleure), Milchlöchli [S.O. 3] (3-8-56); sur parois : $2 \delta^{\circ}$ (Roth, Strinati et Aellen rec.).

- (Vaud), grotte du Pontet 1 [V.D. 29] (24-12-61) ; 1 nymphe (Strinati rec.).

Yougoslavie :

- Novi (17-6-56); sur Rhinolophus euryale : 2 nymphes (B. Djulic rec.) (5).

Grèce :

- Rentina (24-4-55), petites grottes [ $\left.\mathrm{n}^{\circ} 1717\right]$; sur Rhinolophus ferrumequinum : 1 nymphe (Coiffait et Strinati rec.) (6).

Turquie :

- Environs de Silé (29-4-55), grotte Satzmal Magarasi [ $\left.\mathrm{n}^{\circ} 1707\right]$; sur $R$. ferrumequinum: 1 nymphe; sur $R$. hipposideros: 1 nymphe, 10 larves; sur $R$. euryale: 1 , 3 nymphes, 3 larves ; sur Miniopterus schreibersi : 3 larves (C. et S. rec.) (6).

(3) Il s'agit, d'après Hoogstraal (in litt. 27-4-66) d'A. transgariepinus.

(4) Nous tenons à remercier les collecteurs de ce matériel et en particulier MM. Dr Aellen, $\mathrm{D}^{\mathrm{r}}$ Balazuc, Balliot, Bou, Bouillon, Carrère, Coiffait, Constant, $\mathrm{D}^{\mathrm{r}}$ Leclercq, Legrand, Lucas, Magné; Menu, Mesnier, Salvayre, Strinati.

(5) Ce matériel nous a été confié par le $\mathrm{D}^{x} \mathrm{~V}$. Aellen que nous remercions très vivement. Le Matricule entre crochets (pour les exemplaires suisses) correspond au numéro de la cavité in Aellen et Strinati, 1962. 
- Environs de Hacilli (30-4-55), grottes Yassikaya [n $\left.{ }^{\circ} 1709\right]$; sur $R$. ferrumequinum : 2 \&, 5 nymphes, 1 larve (C. et $S$. rec.) (6).

- Inkaya Köyü, près Bursa (11-5-55), grotte Suini [n $\left.{ }^{\circ} 1710\right]$; sur Miniopterus schreibersi : 1 larve (C. et S. rec.) (6).

\section{Afghanistan :}

- Kandahar (14-4-58), grotte Chamchir ; sur Miniopterus schreibersi pallidus : 2 nymphes (K. Lindberg rec.) (5).

Ces captures complètent certains points de distribution ou confirment la présence normale de cette tique.

A notre connaissance, $I$. vespertilionis est nouveau pour la Corse, mais il était déjà connu de Sardaigne (Hoogstraal, 1956).

Il était déjà mentionné de Suisse par Arthur (1956), de Yougoslavie par Neumann (1916) et Oswald (1939), de Grèce par divers auteurs dont Schulze (1936 in Arthur 1956) et Arthur (1956), de Turquie par Arthur (1956). Le matériel en provenance de ces régions est toutefois extrêmement pauvre et c'est pourquoi nous avons jugé bon de publier ces déterminations.

L'Afghanistan par contre n'est mentionné à notre connaissance par aucun des auteurs ayant fait une revue récente de la répartition de l'espèce.

\section{$2^{\circ}$ IXODES SIMPLEX SIMPLEX Neumann, 1906}

Longtemps mal connue, cette tique fut réétudiée par Arthur (1956) et le mâle décrit par nous-même (1962). Depuis cette publication, nous avons eu en mains un assez riche matériel de cette intéressante espèce. Nous en profitons pour compléter la répartition de cette tique et tenter de donner quelques aperçus sur sa spécificité. Quelques points de sa biologie figureront dans une note ultérieure.

\section{Synonymie}

En 1955, Emtchuk (7) décrit $I$. pospelovae. Cette tique, dont seule la femelle est connue, a été capturée sur Myotis oxygnathus, en bordure des Carpathes. D'après la description et les dessins donnés par l'auteur, nous pensons qu'il s'agit d'Ixodes simplex simplex.

En 1958, Babos et Janisch décrivent de Hongrie Ixodes chiropterorum d'après un matériel comprenant femelles, nymphes et larves prélevées sur Miniopterus schreibersi et accessoirement Myotis nattereri et Myotis sp: Cette espèce est maintenue par Babos (1964) dans sa « Faune des Tiques d'Europe Centrale ». Nous pensons, au vu

(6) Matériel également adressé par le $\mathrm{D}^{\mathrm{r}}$ Aellen et récolté par la Mission Coiffait-Strinati (1955); les numéros entre crochets sont ceux qui figurent dans l'article de Strinati (1959).

(7) Nous remercions le $\mathrm{D}^{r}$ I. Grulich de nous avoir signalé cette description. 
de ces publications, qu'il y a lieu de considérer $I$. chiropterorum B. et J. comme synonyme d'I. simplex simplex. Grulich et Zapletal (teste Dusbabek, 1963) identifiaient déjà chiropterorum à pospelovae.

\section{Répartition}

Les révisions concernant cette espèce sont celles de Arthur (1956 et 1965) et de Hoogstraal (1956). La forme type est signalée des pays suivants :

Afrique :

- Congo, Gabon, Afrique du Nord, Soudan, Kenya, Afrique du Sud. Asie :

- Shanghaï, Japon, Palestine.

Australie.

Europe :

- France: Banyuls (Pyr.-Or.), (avril 1937), sur Miniopterus schreibersi, $2 \uparrow$ (Rocky Mountain Laboratory) (Arthur, 1956); Massat (Ariège), grotte du Queire, (18-7-61), sur Miniopterus schreibersi, 1 larve ; id., (17-12-61), sur parois, 1 ơ (Beaucournu, 1962).

- Grèce : (Schulze in Arthur 1956, Loc. ?) ; St-Jean-le-Prodrome, Serrès, 6-51954, sur Miniopterus schreibersi, et Myotis myotis, 2 nymphes (V.A. $\mathrm{n}^{\circ}$ 27) ; grotte de Chrysopighi, Serrès, 1 nymphe libre (V.A. $\mathrm{n}^{\circ} .35$ ) ; grotte Apano Scola, près Naoussa (25-4-54), sur $M$. schreibersi, 4 nymphes, 6 larves (V.A. $\mathrm{n}^{\circ}$ 28); grotte Katapigui, Areopolis (29-11-52), 1 nymphe libre (V.A. $\mathrm{n}^{\circ} 40$ ) (toutes ces références in Arthur, 1956).

Nous pouvons apporter les références suivantes qui mentionnent pour la première fois cette espèce de Corse, Suisse et Turquie.

\section{France :}

- Banyuls (Pyr.-Or.), grotte de Pouade (8) (7-4-47) sur Miniopterus schreibersi, 3 nymphes (coll. Leclercq ; signalées comme 1 . vespertilionis par Leclercq et Theodoridès, 1950); id., (8 et 23-4-59), sur $M$. schreibersi, 1 nymphe, 5 larves (A. Heymer rec.) ;

- Lons-le-Saulnier (Jura), grotte de Macornay (21-5-61), sur $M$. schreibersi ou Myotis myotis, 2 nymphes (P. Constant rec.) (5) ;

- Rancogne (Charente), grotte de Rancogne (2 et 3-3-63), sur $M$. schreibersi, 2 \%; sur parois, 3 ơ (J.C.B. rec.) ;

- La Pradelle-Puylaurens (Aude), grotte des Deux-Entrées (10-4-63), sur M. schreibersi, 1 nymphe (H. Salvayre rec);

- Mosset (Pyr.-Or.), grotte des Inquentades (30-6-63) sur M. schreibersi, 1 nymphe, 2 larves (H. Salvayre rec.) ;

(8) Il s'agit très vraisemblablement du lieu précis d'où proviennent les 2 du Rocky Mountain Laboratory étudiées par Arthur, 1956. 
- Sagone (Corse), grotte des Pigeons (13-7-64) sur $M$. schreibersi, 3 \&, 16 nymphes, 34 larves ; id., (14-7-64), sur parois, 2 ò; id. (30-7-64) sur M. schreibersi, 1 larve ; sur parois, 5 o , 1 q gorgée, 2 nymphes gorgées (J.C.B. rec.).

Suisse :

- Canton de Neuchâtel, grotte du Chemin-de-fer [N.E. 14] (21-5-64) sur Miniopterus schreibersi, 1 nymphe (Aellen rec.) (5).

Grèce :

- Rentina (24-4-55), petites grottes [ $\mathrm{n}^{\circ}$ 1717], sur $M$. schreibersi, 2 larves, (Coiffait et Strinati rec.) (6).

- Près Castoria (28-5-54), Trou de Patarangon, 2 nymphes (libres ?) (K. Lindberg rec.) (5) (cavité habitée par $M$. schreibersi).

\section{Turquie :}

- Inkaya Köyü, près de Bursa (11-5-55), grotte Suini [ $\mathrm{n}^{\circ} 1710$ ], sur $M$. schreibersi, 1 \&, 1 nymphe, 1 larve (Coiffait et Strinati rec.) (6).

D'autre part, en tenant compte de la synonymie proposée ci-dessus, nous rappelons que cette tique est signalée d'Ukraine (sub. nom. I. pospelovae Emtchuk) et de Hongrie (sub. nom. I. chiropterorum Babos et Janisch).

\section{Spécificité}

C'est le seul aspect de sa biologie que nous voudrions approfondir ici. D'après Arthur (1956 et 1965) et Hoogstraal (1956), cette tique serait aussi peu spécifique qu'Ixodes vespertilionis, espèce qui parasite toutes les chauves-souris cavernicoles (Beaucournu, 1961). Nous n'avons aucune expérience en ce qui concerne la zone éthiopienne, mais, pour la zone paléarctique, nous sommes certain que cette tique est un parasite pratiquement spécifique de Miniopterus schreibersi.

Il faut en effet noter d'abord (en ce qui concerne le matériel que nous avons nous-même étudié) que les seuls exemplaires qui ne furent pas capturés avec certitude sur Miniopterus provenaient d'un essaim mixte Miniopterus et Myotis myotis (grotte de Macornay, Jura). D'autre part, nous avons eu fréquemment de la même cavité 1 . vespertilionis et I. simplex (Grotte de Rancogne, Charente; Grotte de Rentina, Grèce ; Grotte Suini, Gr. Satzmal Magarasi, Turquie...) : alors que le premier se trouvait sur diverses espèces y compris Miniopterus, le second ne fut prélevé que sur Miniopterus. Inversement, dans la Grotte des Pigeons (Corse) où vivaient en cohabitation étroite quatre espèces de chauves-souris, dont Miniopterus, seul celui-ci fut trouvé parasité par I. simplex et cela au taux, élevé, de deux par Minioptère. Enfin, dans l'Ouest et le Sud-Ouest de la France où nous avons personnellement récolté et identifié environ 
2.000 tiques cavernicoles du genre Ixodes appartenant à six espèces (Beaucourmu 1962 ; Beaucournu et Matile 1963); I. simplex ne fut trouvé que dans des grottes abritant des Minioptères ; il manque ainsi totalement au nord de la Loire où son hôte fait défaut (9).

\section{$3^{\circ}$ ARGAS (CARIOS) VESPERTILIONIS (Latreille 1802)}

Cette tique commune, mais beaucoup moins fréquente sous terre qu'Ixodes vespertilionis est abondante sur les chauves-souris capturées dans les abris épigés.

Nous en apportons que!ques nouvelles captures (10).

\section{France :}

- Guipry (Ille-et-Vilaine) (10-9-61), sur Pipistrellus pipistrellus, 2 larves (G. Lelièvre rec.); Vassens (Aisne), Carrière souterraine (28-1-61), sur Myotis dasycneme, 1 larve (H. Menu rec.) (5) ; Cornillé (M.-et-Loire), Carrière souterraine $\mathrm{n}^{\circ} 1$ (16-2-64), sur Plecotus austriacus, 2 larves (J.C.B. rec.) ; Tour-du-Valat (Bouches-du-Rhône) (7-8-51), sur Eptesicus serotinus, 3 larves, (R. Levêque rec.) (5).
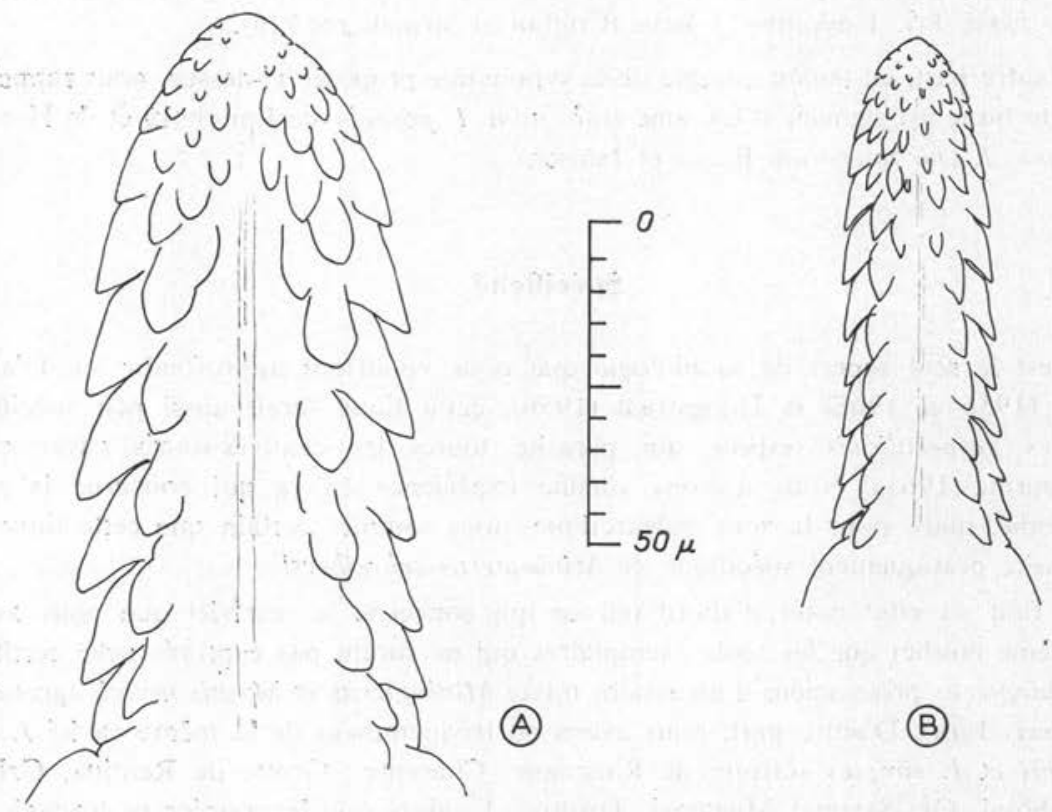

Hypostomes larvaires: A) Argas transgariepinus White, Sempach (Suisse).

B) Argas vespertilionis (Latreille), même origine.

(9) La capture de cette chauve-souris dans le Loir-et-Cher (Caubère, 1952, Mammalia, 16, 256) est erronée (Caubère, in litt., 1-6-63).

(10) De nombreux prélèvements provenant de régions ou d'hôtes déjà mentionnés dans notre note de 1961 sont omis ici. 
Suisse :

- Genève (Mus. Hist. Nat.), s.d., sur Nyctalus noctula, plusieurs larves (5) ; id. (31-8-56) sur Pipistrellus pipistrellus, plusieurs larves (Aellen rec.) ; Commugny (Vaud) (8-55), sur Pipistrellus nathusii, plusieurs larves (Steffen et Aellen rec.) ; Sempach (Lucerne) (7-7-63), sur $P$. nathusii, 2 larves (Aellen rec.).

\section{$4^{\circ}$ ARGAS (SECRETARGAS) TRANSGARIEPINUS White, 1846}

Cette tique, encore assez mal connue quant à sa répartition et à sa biologie, est inféodée aux Chiroptères. Elle est morphologiquement très dissemblable de $A$. vespertilionis; signalons essentiellement sa taille beaucoup plus grande, son corps ovoïde et non sub-circulaire.

D'après Hoogstraal (in litt., 25-XI-64, et 1952, 1954), elle est connue « de l'Espagne à la Grèce, de Palestine, d'Afrique du Nord (Egypte) et d'Afrique du Sud ». Starkoff (1958) la cite d'Italie. Elle n'est pas connue, pour autant que nous le sachions, de France. Nous apportons la référence suivante, nouvelle pour le pays d'origine.

Suisse :

- Sempach (Lucerne) (7-7-63), sur Pipistrellus nathusii, 1 larve (Aellen rec.) (11).

Nous donnons ci-après les dessins des hypostomes de cette larve et d'une larve d'A. vespertilionis de même origine, figurés à la même échelle.

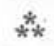

Il est certain que ces données sont encore fragmentaires. Certaines espèces vont encore enrichir un jour ou l'autre notre faune française (par exemple Argas transgariepinus). D'autre part, nous pensons qu'il reste encore de nouvelles formes à décrire et que, surtout, de patientes observations sont à faire pour élucider la biologie de ces ectoparasites, biologie pratiquement inconnue à l'heure actuelle.

\section{Résumé}

Ce travail donne de nouvelles précisions sur la répartition d'Ixodes vespertilionis et Ixodes simplex simplex. Ce dernier est en particulier cité pour la première fois de Corse, de Suisse et de Turquie.

D'autre part, l'auteur propose de placer en synonymie d'I. s. simplex, I. chiropterorum Babos et Janisch et $I$. pospelovae Emtchuk.

De nouvelles références sont données concernant la répartition d'Argas vespertilionis et d'A. transgariepinus (ce dernier cité pour la première fois de Suisse).

(11) Ce spécimen a été identifié par le $\mathrm{D}^{r}$ Hoogstraal que nous remercions vivement de son obligeance. 


\section{Bibliographie}

Aellen (V.) et Strinati (P.), 1962. - Nouveaux matériaux pour une faune cavernicole de la Suisse. Rev. Suisse Zool., 69, 25.

ARThUR (D. R.), 1956. - The Ixodes ticks of Chiroptera (Ixodoidea : Ixodidae). J. Parasit., $42,180$.

-, 1965. - Ticks of the genus Ixodes in Africa. The Athlone Press, London, 348 pp.

BAbos (S.), 1964. - Die Zecken Mitteleuropas, Akademiai Kiado, Budapest, 410 pp.

- et JANISCH (M.), 1958. - Ixodes chiropterorum sp. n. eine neue Zeckenart in Hungarn. Acta Vet. Acad. Sci. Hung., 8, 389.

Beaucournu (J.-C.), 1961. - Ectoparasites des Chiroptères de l'Ouest de la France. $1^{\text {ro }}$ partie : Ixodoïdés, Cimicidés et Nyctéribiidés. Bull. Soc. Scient. Bretagne, 36, 315.

—, 1962. - Description du mâle d'Ixodes simplex simplex Neumann, 1906, parasite de chiroptères. Bull. Soc. Zool. France, 87, 127.

- et Matile (L.), 1963. - Contribution à l'inventaire faunistique des cavités souterraines de POuest de la France. 3 : Liste des Espèces; Bibliographie. Ann. Spéléo., 18, 519.

Colas-Belcour (J.), 1929. - Présence d'Ornithodorus coniceps en Tunisie. Arch. Inst. Pasteur Tunis, 18, 265.

DusbabeK (F.), 1963. - Parasitische Fledermausmilben der Tschechoslowakei IV. Acta Soc. Ent. Cechoslov., 60, 332.

Емтсник (Е. M.), 1960. - [Faune de l'Ukraine, vol. 25. Tiques Ixodoidea, $\mathrm{n}^{\circ}$ 1]. Acad. Sc. d'Ukraine, Kiev, p. 77 (en Ukrainien).

Hoogstraal (H.), 1952. - Notes on Egyptian ticks (Ixodoidea). 1. The genus Argas (Argasidae) in the Cairo area. Proc. Egypt. Acad. Sci., 7, 114.

- 1954. - A preliminary, annotated list of ticks (Ixodoidea) of the Anglo-Egyptian Sudan. J. Parasit., 40, 304.

-, 1956. - African Ixodoidea, vol. 1: Ticks of the Sudan. Res. Report., Dept. of Navy, $1.100 \mathrm{pp}$.

LECLERQ (M.) et THÉODORIDÈs (J.), 1950. - Some ectoparasites of birds and mammals observed recently in France. Ent. Month. Mag., 86, 74.

Neumann (L. G.), 1897. - Révision de la famille des Ixodidés; $2^{\circ}$ mémoire. Mém. Soc. Zool. France, 10, 324.

—, 1906. - Notes sur les Ixodidés. Arch. Parasit., 10, 195.

—, 1911. - Ixodidae in «das Tierreich », 26, Berlin, Friedlander éd., 169 pp.

-, 1916. - Biospeologica, 37 : Ixodidei (Acariens), $1^{\text {re }}$ partie. Arch. Zool. exp. gen., 55, 517.

Oswald (B.), 1939. - On Yugoslavian (Balkan) ticks (Ixodoidea). Parasitology, 31, 271.

Starkoff (O.), 1958. - Ixodoidea d'Italia. Il pensiero scientifico, Roma, 385 pp.

Strinati (P.), 1959. - Mission Coiffait-Strinati en Macédoine, Grèce et Turquie (avril-mai 1955). Chiroptera. Mammalia, 23, 72.

[Laboratoire de Parasitologie et Zoologie appliquée

de la Faculté de Médecine et de Pharmacie de Rennes (Professeur: J.-M. DoBY)] 